\title{
Ausblicke auf die fallpauschalierte Spitalfinanzierung im Jahr 2013
}

\section{Dominik Franz, \\ Holger Bunzemeier, \\ Wolfgang Fiori, \\ Norbert Roeder}

DRG-Research-Group, Universitätsklinikum Münster (Leiter: Prof. Dr. N. Roeder)
Korrespondenz:

Dr. med. Dominik Franz Geschäftsbereich Medizinisches Management -

Medizincontrolling, DRG-Research-Group Universitätsklinikum Münster Domagkstrasse 20 D-48129 Münster Tel. 0049251 83-52023 Fax 0049251 83-52019 ukmuenster.de

\section{Einleitung}

Der Systemwechsel in der Vergütung stationärer Spitalleistungen ist erfolgt. Seit dem 1.1.2012 ist die SwissDRG Version 1.0 die Grundlage der Vergütung vollstationärer somatischer Fälle in Akutspitälern und Geburtshäusern [1]. Auch die für 2013 weiterentwickelte Version 2.0 wurde bereits veröffentlicht [2]. Nach den ersten Anwendungserfahrungen stellen sich Leistungserbringer und Kostenträger wichtige Fragen. Kann mit der vorhandenen Datenqualität und der Methodik der Kostenkalkulation eine sachgerechte Vergütung gewährleistet werden? Wie soll mit hochkomplexen medizinischen Bereichen umgegangen werden, deren Fallzahl sehr gering ist? Ist der CHOP-Katalog ausreichend differenziert, um Ressourceninformationen für die Kostenkalkulation und die Fallgruppierung bereit zu stellen?

Dieser Beitrag beschreibt die wesentlichen Weiterentwicklungen der SwissDRG Version 2.0 und stellt noch zu lösende Problembereiche dar.

\section{Die SwissDRG Version 2.0}

Die Planungsversion 2012/13 der Version 2.0 wurde im August 2012 veröffentlicht [2]. Sie ermöglicht eine Gruppierung von Daten des Jahres 2012. Bei der Konzeption konnte die SwissDRG AG noch von den Erfahrungen des deutschen DRG-Instituts InEK profitieren. Tabelle 1 stellt die Versionen 1.0 und 2.0 vor.

\section{Diagnose- und Prozedurenklassifikation}

Für die Datenerhebung 2012 gelten die ICD-10-GMVersion 2010 und die CHOP-Version 2012. Wesentliche Veränderungen der ICD-10-GM-Versionen 2009 und 2010 gegenüber 2008 sind z.B. die Einführung neuer Kodes für das diabetische Fusssyndrom und Neuordnungen von ICD-Kodes für die akute Appendizitis, für chronisch entzündliche Darmerkrankungen, Dekubitalgeschwüre sowie die chronische Nierenkrankheit.

Grundlage der Weiterentwicklung des CHOP 2011 zur Version 2012 sind die Ergebnisse des SwissDRG-Antragsverfahrens 2010. Neue CHOP-Kodes betreffen beispielhaft gefäss- und thoraxchirurgische Operationen, kardiologische Interventionen, bildgebende Verfahren sowie die Erweiterung von Komplexbehandlungen

Die Veränderungen der ICD-10-GM und des $\mathrm{CHOP}$ werden zu einer klassifikatorisch differenzier-

\section{Zusammenfassung}

Der Wechsel zum SwissDRG-System ist erfolgt. Auch die ab 2013 geltende SwissDRG-Version 2.0 liegt bereits vor. Die Zahl der DRGs in der Version 2.0 sinkt um 61. Der Rückgang betrifft v. a. hochkomplexe Fälle aus den Bereichen Langzeitbeatmung, Neonatologie und Onkologie. Die Version 2.0 erhöht die Sachgerechtigkeit der Leistungsabbildung schrittweise gegenüber der Vorversion im Rahmen der vorhandenen Möglichkeiten. Dennoch ist die bisher erreichte Systemgüte weiter optimierbar und auch in der Version 2.0 noch nicht ausreichend, um die Spitalleistungen allein auf der Basis Schweizer Daten sachgerecht zu kalkulieren. Auf dem Weg zur Zielerreichung, die der Gesetzgeber mit der SwissDRGEinführung vorgab, kann die Version 2.0 nur eine Zwischenlösung sein. Die Folgeversion 3.0 wird u. a. auf erheblichen Veränderungen der ICD-10-GM- und CHOP-Strukturen aufbauen können, die zu klassifikatorisch differenzierteren Möglichkeiten der Diagnose- und Prozedurenkodierung führen werden, so dass zukünftig mit weiteren Steigerungen der Sachgerechtigkeit der Leistungsabbildung zu rechnen sein wird.

teren Diagnose- und Prozedurenkodierung führen. Insbesondere beim CHOP ergibt sich daraus z. B. für gefässchirurgische Verfahren eine spezifischere Darstellung des Ressourcenaufwandes [3]. Dennoch wird es auch weiterhin noch nicht möglich sein, mittels CHOP-Kodes z. B. teure Medikamente fallbezogen zu kodieren, so dass separate Listen die Erfassungen ergänzen müssen (s. u.).

\section{Die Tarifstruktur der SwissDRG Version 2.0}

Die Version 2.0 enthält 61 Fallpauschalen weniger als die Vorversion. Dieser Rückgang betrifft ausschliesslich bewertete DRGs, v. a. in den Hauptdiagnosekate- 
gorien (MDC) der PräMDC (hochkomplexe Fälle mit Transplantationen oder Langzeitbeatmungen), der MDC15 Neugeborene und der MDC17 Hämatologische und solide Neubildungen (Tabelle 1).

\section{Kann mit der vorhandenen Datenqualität und der Methodik der Kostenkalkulation eine sachgerechte Vergütung gewährleistet werden?}

Beispielhaft sei auf den Rückgang der Zahl der DRGs in der PräMDC eingegangen. Die PräMDC umfasst klinisch und ökonomisch sehr komplexe Fallgruppen, die jedoch nur in sehr geringer Zahl vorkommen (z. B. Langzeitbeatmungen $>1.000 \mathrm{~h}$ ). Eine statistisch tragbare Kalkulation anhand von Schweizer Daten ist durch die geringe Fallzahl erschwert. Auch zukünftig wird keine Fallzahlerhöhung erwartet [4]. Bei einer zu kleinen Stichprobe besteht die Gefahr, dass jährlich stark schwankende Kostengewichte resultieren, die nicht den tatsächlichen landesdurchschnittlichen Kosten im Abrechnungsjahr entsprechen $[5,6]$. Für die Version 1.0 ist davon auszugehen, dass ein Großteil der Kostengewichte in der PräMDC und der MDC15 aus helvetisierten deutschen Kostengewichten hervorging.

In der Version 2.0 wurde die Staffelung der DRGrelevanten Beatmungszeiten reduziert. Dies senkt die Bewertungsspannbreite und die Höhe der Kostengewichte in der PräMDC. Eine vergleichbare Massnahme erfolgte für Neugeborene mit geringem Geburtsgewicht $(<1.000 g)$. Für auf hochkomplexe Behandlungsfälle spezialisierte Spitäler (z. B. Universitätsspitäler) kann eine Casemixreduktion und je nach Kostensituation und Basisfallwert ggf. auch eine Unterfinanzierung resultieren. Allerdings erhöht die DRG-Kondensation die Fallzahlen in den verbleibenden DRGs, so dass die Voraussetzungen für eine eigenständige Schweizer Kostenkalkulation steigen

\begin{tabular}{|c|c|c|}
\hline \multicolumn{3}{|c|}{$\begin{array}{l}\text { Swiss-DRG-Versionsvergleich [4]. Anm.: Die Angaben zu den helvetisierten und den auf } \\
\text { Schweizer Daten errechneten Kostengewichten stammen von einer Folie der SwissDRG AG. } \\
\text { Beide Angaben für 2012/13 wurden übernommen, obwohl die Summe beider Zahlen nicht } \\
975 \text { ergibt. }\end{array}$} \\
\hline & Version 1.0 & Version 2.0 \\
\hline n DRGs & 1.052 & $991(-5,8 \%)$ \\
\hline n bewertete DRGs & 1.036 & $975(-5,9 \%)$ \\
\hline n unbewertete DRGs & 16 & $16( \pm 0)$ \\
\hline $\begin{array}{l}\text { n auf Schweizer Daten kalkulierte } \\
\text { DRGs }\end{array}$ & 804 & 812 \\
\hline n helvetisierter Kostengewichte & 232 & 161 \\
\hline Zusatzentgelte (ZE) & \multicolumn{2}{|c|}{ In beiden Versionen 3 bewertete und 2 unbewertete ZE } \\
\hline
\end{tabular}

(Tabelle 1). Dies ist vorteilhaft, weil die Versorgungsstrukturen im Vergleich zu Deutschland abweichen [4].

In beiden SwissDRG-Versionen existieren unverändert drei bewertete (verschiedene Dialysen) und zwei unbewertete («KunstherZ»-Versorgung bzw. Blutgerinnungsfaktoren für Hämophiliepatienten) $\mathrm{Zu}$ satzentgelte. Zusatzentgelte können zusätzlich zu einer SwissDRG-Pauschale abgerechnet werden und vergüten aussergewöhnlich hohe Kosten für bestimmte Behandlungen, die nicht auf bestimmte DRGs beschränkt sind. Bewertete Zusatzentgelte sind mit einem schweizweit einheitlichen Frankenbetrag bewertet. Für unbewertete Zusatzentgelte muss die Vergütungshöhe spitalindividuell verhandelt werden [4]. Auch die Version 2.0 sieht keine Zusatzentgelte für teure Medikamente (z. B. Antikörper, Antimykotika usw.) oder teure Medizinprodukte (z. B. spezielle Implantate, Prothesen, usw.) vor. Dies kann z. B. für komplexe Fallkonstellationen insbesondere in der Onkologie, Rheumatologie, Orthopädie und Unfallchirurgie, Gefäßchirurgie sowie Neurologie problematisch sein. Hier können teure Medikamente/Implantate zu einer erheblichen Leistungs- und Kostenheterogenität führen, die allein über DRGs nicht sachgerecht abgebildet wird (s. u.) Damit wird ein ökonomischer Anreiz gesetzt, Indikationen restriktiver zu stellen bzw. bei Implantaten qualitativ minderwertigen Produkten Vorrang einzuräumen.

Neben DRGs und Zusatzentgelten sind auch Innovationsentgelte ein wichtiger Baustein der Spitalfinanzierung, auf den in diesem Beitrag nicht vertieft eingegangen werden kann. Das durch die SwissDRG AG vorgestellte Verfahren zur Finanzierung von Innovationen erlaubt frühzeitig spitalindividuelle Verhandlungen, sieht jedoch einen mindestens 5-jährigen Umsetzungszeitraum vor.

\section{Die Kostenkalkulation}

Unterschiedlich aufwändige Behandlungsfälle lassen sich nur dann in einem DRG-System abbilden, wenn die Kosten in den Spitälern sachgerecht kalkuliert werden. Dies gilt v. a. für die fallbezogene Zuordnung von hohen Einzelkosten [5, 6]. Auch in der Version 2.0 ist die Güte der Fallkostenkalkulation noch nicht ausreichend genug, um die Leistungen in den Spitälern sachgerecht und allein auf der Basis Schweizer Daten zu kalkulieren [4].

Um die Datenqualität zu erhöhen, wurden für die Erhebung 2012 Listen für in der Medizinischen Statistik erfassbare Medikamente, teure Verfahren und Implantate vereinheitlicht und eine Mindestschwelle für teure Medikamente definiert [7]. Für die Datenerhebung 2013 ist die Unterscheidung der Kostenstellen «OP-Saal» und «Anästhesie» vorgesehen [4] und ab der Datenerhebung 2014 wird REKOLE ${ }^{\circledast}$ [8] verbindlich als einheitliche Methode für die Erstellung der Kostenträgerrechnung vorgeschrieben [7]. Dies sind wichtige Schritte, um sachgerechtere Kal- 
kulationen auf Basis von Schweizer Daten zu ermöglichen.

Diese Veränderungen sind notwendig, da der Rückgriff auf helvetisierte deutsche Kostendaten methodisch immer problematischer wird, je eigenständiger sich das SwissDRG-System weiterentwickelt $[5,6]$. Insbesondere da an der Datenerhebung 2013 alle Schweizer Akutspitäler obligatorisch auch mit Kostendaten teilnehmen werden, besteht für die SwissDRG AG die große Herausforderung, trotz notwendiger stringenterer Kalkulationsvorgaben, den Spitälern die Teilnahme an der Kalkulation zu ermöglichen. Deutsche Erfahrungen zeigen, dass die Kalkulation komplexer medizinischer Leistungen einen erheblichen Aufwand und erfahrene Krankenhausmitarbeiter erfordert [5, 6]. Die Vollerhebung erhöht zwar die Fallzahlen, eine unzureichende Datenqualität kann jedoch die Qualität der Kalkulation negativ beeinflussen.

\section{Neben DRGs und Zusatzentgelten sind auch Innovationsentgelte ein wichtiger Baustein der Spital- finanzierung.}

\section{Individuelle Basisfallwerte}

Durch die Verhandlung spitalindividueller Basisfallwerte bestehen für die Leistungserbringer weiterhin Spielräume, um u. a. Unterfinanzierungen durch noch nicht ausreichend sachgerechte Tarifstrukturen auszugleichen. Allerdings reduzieren verhandelbare Basisfallwerte auch den ökonomischen Druck auf die Leistungserbringer, welcher im Sinne der Hebung von durch den Gesetzgeber vermuteten Effizienzreserven in den Spitälern ein wesentliches Ziel der SwissDRG-Einführung war [3, 5, 6]. Um Planungssicherheit für die Spitäler zu erreichen, bedarf es jedoch vorrangig sachgerechter Lösungen innerhalb der Tarifstruktur. In der Diskussion um unterschiedliche Basisfallwerte für verschiedene Versorgungsstufen sollte der Einfluss auf den Wettbewerb der Spitäler berücksichtigt werden [3].

\section{Fazit}

Das SwissDRG-System entwickelt sich im Sinne eines «lernenden Systems» weiter. Die Version 2.0 verbessert die Sachgerechtigkeit der Leistungsabbildung gegenüber der Vorversion im Rahmen der vorhandenen Möglichkeiten [4]. Sie baut auf einer verbesserten Basis aus Leistungsdaten (differenziertere Diagnosen und Prozeduren) und Kostendaten (einheitliche Medikamentenliste u. a.) auf. Diese Grundlage wird durch gezielte verbindlichere Vorgaben (s. o.) zukünftig weiter verbessert. Eine enge Zusammenarbeit zwi- schen InEK und SwissDRG AG erscheint in diesem Zusammenhang sehr nützlich. Die Reduzierung der Zahl an SwissDRGs - die Kondensation der Tarifstruktur - betrifft Bereiche, die massgeblich durch helvetisierte deutsche Daten strukturiert wurden. Die geringen Fallzahlen, die Schwächen der Kostenkalkulation und z.T. unterschiedliche Versorgungsstrukturen machen eigenständige Schweizer Lösungen erforderlich. Dieser Prozess hat begonnen und wird sich fortsetzen.

Auf dem Weg zur Erreichung der Ziele, die der Schweizer Gesetzgeber mit der Einführung des SwissDRG-System setzte, kann die Version 2.0 erwartungsgemäss nur eine Zwischenlösung sein. Sowohl innerhalb der Tarifstruktur als auch außerhalb müssen zukünftig weitere wesentliche Schritte unternommen werden, um auf der Basis von Schweizer Leistungsund Kostendaten eine sachgerechte Fallkostenkalkulation verbunden mit einer hohen Abbildungsqualität durch das SwissDRG-System zu erzielen. Die Folgeversion 3.0 wird $u$. a. auf erheblichen Veränderungen der ICD-10-GM- und CHOP-Strukturen aufbauen können, so dass zukünftig mit weiteren Steigerungen der Sachgerechtigkeit der Leistungsabbildung zu rechnen sein wird.

\section{Literatur}

1 SwissDRG AG: Fallpauschalen-Katalog SwissDRG Version 1.0. Download: www.swissdrg.org/de/07 casemix_office/SwissDRG_System_10.asp?navid=29

2 SwissDRG AG: Fallpauschalen-Katalog SwissDRG Version 2.0 Download: www.swissdrg.org/de/07 casemix_office/SwissDRG_System_20.asp?navid=25

3 Meyer B, Ingenpass P. SwissDRG-Version 2.0 Einschätzungen der FMH und Empfehlungen. Schweiz Ärztezeitung. 2012; 93(22):797-8.

4 SwissDRG AG: Folien der SwissDRG Infoveranstaltung «SwissDRG-Version 2.0/2013Z, Bern 13.6.12. Download unter: www.swissdrg.org/de/ 06_swissdrg_ag/Veranstaltungen/20120613_Version_ 2.0_Homepage.pdf

5 Franz D, Bunzemeier H, Fiori W, Wenke A, Helling J, Brüning K, et al. Die SwissDRGs - ein Zwischenbericht zur Einführung der fallpauschalierten Spitalfinanzierung auf der Grundlage deutscher DRG-Strukturen (I) Das Krankenhaus, 2010;11:1074-80.

6 Franz D, Bunzemeier H, Fiori W, Wenke A, Helling J, Brüning K, et al. Die SwissDRGs - ein Zwischenbericht zur Einführung der fallpauschalierten Spitalfinanzierung auf der Grundlage deutscher DRG-Strukturen (II). Das Krankenhaus 2010;12:1196-204.

7 SwissDRG AG: Umsetzung der Maßnahmen zur Verbesserung der Datenqualität. Version 1.0 / 5.12.2011. Download unter: www.swissdrg.org/assets/ pdf/Erhebung2012/Umsetzung_Massnahmen_ Datenqualitaet.pdf

8 REKOLE - betriebliches Rechnungswesen im Spital. H+ Die Spitäler der Schweiz (Hrsg.) ISBN 978-3-9523416-0-5. 